\title{
MedienPädagogik
}

Zeitschrift für Theorie und Praxis der Medienbildung

Themenheft Nr. 42: Optimierung in der Medienpädagogik.

Forschungsperspektiven im Anschluss an den 27. Kongress der DGfE

Herausgegeben von Patrick Bettinger, Klaus Rummler und Karsten D. Wolf

\section{Die Optimierung von Medienentscheidungen in der Unterrichtsplanung - eine Utopie?}

Simon Küth, Daniel Scholl, Christoph Schüle und Kathrin Rheinländer

\begin{abstract}
Zusammenfassung
Der Beitrag zielt auf eine mediendidaktische Interpretation der Optimierungsidee und diskutiert die Verknüpfung einer kognitionspsychologischen und informationstechnologischen Sichtweise. Ausgangspunkt ist das zentrale mediendidaktische Prinzip, das besagt, dass optimale Medienentscheidungen bei mediengestützten Lernangeboten in Wechselwirkungen mit anderen Planungsbereichen stehen sollten, zum Beispiel mit den Lernvoraussetzungen, -zielen oder-inhalten. Die Konsequenz dieses Interdependenzprinzips ist, wechselwirkende Medienentscheidungen mit den weiteren Planungsentscheidungen und -voraussetzungen bereits in der Unterrichtsplanung treffen zu müssen, die zu einem möglichst stimmigen Unterrichtsentwurf führen, dessen Qualität dann im Kreislauf von Planung, Durchführung und Analyse der Lernangebote stetig weiter zu entwickeln ist. Im vorliegenden Beitrag wird dieses mediendidaktische Prinzip aufgegriffen und zunächst aus der Perspektive der Cognitive Load-Theorie gefragt, ob das Optimum einer interdependenten Unterrichtsplanung die Planenden - massgeblich Planungsnovizinnen und -novizen - kognitiv überfordert und deshalb eine Utopie bleiben muss. Anschliessend wird eine technologiebasierte Lerngelegenheit - eine Planungssoftware - vorgestellt, die das kognitiv entlastende Erlernen interdependenter Planungsentscheidungen unterstützt und einen möglichen Weg aufzeigt, das Treffen interdependenter Planungsentscheidungen selbst für Planungsnovizinnen und -novizen realistisch werden zu lassen.
\end{abstract}

Optimizing media decisions in lesson planning - a utopia?

\begin{abstract}
This article offers a media-didactic interpretation of the optimization idea which is discussed by combining perspectives from cognitive psychology and information technology. A central media-didactic principle states that decisions for optimized mediasupported learning interact with, for example, analysed individual preconditions, decisions on goals or content. The consequence of this interdependency principle is having to make interactive media decisions in line with all other planning decisions and requirements as early as during lesson planning. The result is supposed to be a lesson design that is as coherent as possible, the quality of which must then be continuously
\end{abstract}


developed in an ongoing cycle of planning, implementation and analysis of lesson and instruction. In the present article the perspective of cognitive load theory is used on this media-didactic principle to question whether optimized interdependent lesson planning fails due to the cognitive architecture of the planners and must therefore remain a utopia. A technology-based learning opportunity - a planning software - is introduced which supports learning of interdependent planning decisions by relieving cognitive load. This software points towards a potential way to make interdependent planning decisions, even for beginners.

\section{1. $\quad$ Einleitung}

Das Treffen von Medienentscheidungen zu mediengestützten Lernangeboten gehört zu den zentralen Aufgaben der Unterrichtsplanung (Kerres und Kalz 2003). Da Medien allein allerdings nur einen eher geringen Einfluss auf den Lernerfolg haben (Hattie 2015; Russell 1999; Schulmeister 2007), müssen diese Entscheidungen didaktisch sinnvoll sein und beispielsweise auf durchdachten Beziehungen der Medien zu Lernvoraussetzungen, -zielen oder -inhalten beruhen (Petko 2020; de Witt und Czerwionka 2013). Aus mediendidaktischer Perspektive sind solch durchdachte Medienentscheidungen deshalb unverzichtbar für möglichst effektive mediengestützte Lernangebote - sei es mit dem ausdrücklichen Ziel, die Medienbildung zu fördern, oder sei es eher instrumentell zur Unterstützung der unterrichtlichen Kommunikationsprozesse -, denn gerade Fehler in der Medienplanung können im Unterricht kaum kompensiert werden (Kerres 2005).

Mit diesen angedeuteten Anforderungen an die Planung des Medieneinsatzes sind Medienentscheidungen der übergreifenden Idee der Optimierung (Balandis und Straub 2018) verpflichtet, die für die Unterrichtsplanung insgesamt gilt: Immer geht es bei dieser Planung um eine "Optimierung der Entscheidungsprozesse» (Wengert 1989, 6), wobei das Planen Tätigkeiten umfasst, die «dazu dienen, [...] unterrichtliches Handeln optimal zu organisieren» (Bromme und Seeger 1979, 4) und einen «möglichst optimierten Umgang mit der geringen Prognostizierbarkeit pädagogischer Prozesse» (Gassmann 2013, 116) zu gewährleisten.

In der Konkretisierung dieser Optimierungsidee lassen sich in mediendidaktischen Planungsansätzen deutliche Bezüge zur Allgemeinen Didaktik und dem Planungsprinzip der Interdependenz - der Forderung nach widerspruchsfreier Wechselwirkung sämtlicher Planungsentscheidungen -, das seit der Berliner Didaktik (z. B. Heimann [1962] 1976) als eine bedeutsame Norm für Medienentscheidungen gilt, erkennen. Die Berliner Didaktik hatte angenommen, dass sich die Planung von Unterricht in die Struktur- und in die Verlaufsplanung unterscheiden lasse. Während es in der Verlaufsplanung darum gehe, den folgenden Unterricht zu antizipieren und eine Abfolge spezifischer Lehr-Lernhandlungen festzulegen, sollten in der 
vorangehenden Strukturplanung die sogenannten formalen Konstanten, als deren inhaltliche Variation sich ein jeder Unterricht zeige, in ihren Wechselbeziehungen reflektiert werden (Schulz 1972). Diese formalen Konstanten, die in der Berliner Didaktik die Grundlage für ein Entscheidungsmodell der Unterrichtsplanung bilden, lassen sich in vier Entscheidungsfelder (Intentionalität, Thematik, Methodik, Medienwahl) und zwei Bedingungsfelder (Anthropogene Voraussetzungen, Sozial-kulturelle Voraussetzungen) unterteilen (Schulz 1972). Die Aufgabe der Planenden sei es, Unterricht als Gesamtproblem zu betrachten und die strenge Interdependenz des Verhältnisses der Entscheidungen und Bedingungen in den sechs Feldern zu reflektieren (Heimann [1962] 1976). Optimal im Sinne dieser Reflexion seien die Planungs- und damit auch Medienentscheidungen dann, wenn der Unterrichtsentwurf kohärent sei.

Mit diesem Prinzip der Interdependenz hat die Berliner Didaktik eine relative Auffassung von Optimierung der Medienentscheidungen begründet, die die Strukturqualität mediengestützter Lernangebote betrifft. Ob Strukturqualität gegeben ist und die angenommene Kohärenz der Planung tatsächlich zu einem lernwirksamen Angebot geführt hat, lässt sich erst im Anschluss an die Umsetzung des mediengestützten Lernangebots durch den Abgleich mit dem Plan feststellen, zum Beispiel durch die Prüfung der erreichten Lernziele vor dem Hintergrund der Passgenauigkeit der Medienfunktionen zu den angenommenen lernbereichsspezifischen Voraussetzungen der Lernenden. Die Optimierung von Planungsentscheidungen stellt sich damit in der Berliner Didaktik - einem prinzipiellen Merkmal der Optimierungsidee entsprechend - als «ein relativer, nicht abzuschließender Prozess» (Bellmann, Caruso, und Kleinau 2020, 2) dar.

Im Folgenden soll diese mediendidaktische Auffassung von Optimierung mit ihren Bezügen zur Allgemeinen Didaktik aufgegriffen werden. Dabei zielt der Beitrag nicht darauf, diese Optimierungsidee selbst zu hinterfragen. Vielmehr wird aus einer ergänzenden kognitionspsychologischen Perspektive diskutiert, ob diese Idee, mitsamt den mediendidaktischen Empfehlungen zur interdependenten Medienplanung, an den Grenzen der menschlichen kognitiven Architektur scheitert, die nämlich das Treffen interdependenter Entscheidungen und damit Optimierungsversuche der Strukturqualität mediengestützter Lernangebote verhindern könnte. Ein wenig provokant zugespitzt wird diese Prüfung im Kontext der Frage, ob sich das Prinzip interdependenter Medienentscheidungen als mediendidaktische Optimierungsidee deshalb als Utopie erweise.

Unter Rückgriff auf empirische Befunde zur Unterrichtsplanung wird diese Utopie-Hypothese zunächst hergeleitet, bevor sie genauer aus der Perspektive der Cognitive Load-Theorie (Sweller, van Merriënboer, und Paas 2019, 1998) erklärt wird. Schliesslich wird eine technologiebasierte Lerngelegenheit - eine Planungssoftware - vorgestellt, die Planungsnovizinnen und -novizen beim kognitiv entlastenden 
Erlernen des Treffens interdependenter Medienentscheidungen unterstützen und dabei helfen soll, das Prinzip der Interdependenz bereits ab den ersten Planungsversuchen realistisch zu machen.

\section{Interdependente Medienentscheidungen und die Komplexität der Planungsaufgabe}

Kernannahmen des Planungsprinzips der Interdependenz zur Verknüpfung der einzelnen Planungsentscheidungen in den verschiedenen Planungsbereichen finden sich sowohl in nationalen (Stender 2014) und internationalen (Friesen 2010) allgemeinen Unterrichtsplanungsmodellen als auch in unterschiedlichen, spezifisch mediendidaktischen Ansätzen (z. B. direkt bei Kerres 2000, oder indirekt bei Mayrberger 2019). Ein Blick in empirische Befunde zur Einlösung dieses Prinzips offenbart allerdings, dass gerade Novizinnen und Novizen grundsätzlich Schwierigkeiten mit interdependenten Planungsentscheidungen haben (Koeppen 1998). Speziell Referendarinnen und Referendare sowie Lehrkräfte mit wenig Berufserfahrung konzentrieren sich in ihrer Planung oft ausschliesslich auf einzelne Planungsbereiche (wie z. B. die Inhalte oder die Medien), ohne diese Bereiche miteinander zu verschränken (Seifried 2009). Diese unzureichende Berücksichtigung der Wechselwirkung der Planungsentscheidungen zeigt sich auch daran, dass das Entscheidungsverhalten von unerfahrenen Lehrkräften eher linear ist (Westerman 1991), obwohl ein dynamisches Entscheidungsverhalten dem Prinzip der Interdependenz besser entsprechen würde. Selbst in gemeinsamen Planungsgesprächen unter Studierenden, die nach den Regeln des kollegialen Unterrichtscoachings vonstatten gehen (Kreis und Staub 2017), gelingt eine kognitiv anspruchsvolle Auseinandersetzung durch Verknüpfung der Planungsentscheidungen nur selten, auch nicht nach einer expliziten Intervention (Weitzel und Blank 2019). Übereinstimmend mit diesen Befunden stellt Gassmann (2013) insgesamt fest, dass sich Planungsnovizinnen und -novizen mit der Begründung getroffener Planungsentscheidungen, mit der Anbindung von Inhalten, Zielen, Medien und Methoden an Lernvoraussetzungen der Schülerinnen und Schüler sowie mit der Verknüpfung von Planungsentscheidungen untereinander und mit curricularen Entscheidungen schwertun.

Zumindest für Planungsnovizinnen und -novizen lässt sich deshalb feststellen, dass die mediendidaktische Forderung nach interdependenten Medienentscheidungen keine Entsprechung in der Planungsrealität hat - eine Feststellung, die das Prinzip der Interdependenz möglicherweise als utopische Anforderung für Novizinnen und -novizen erscheinen lässt. Ein solcher ernüchternder Befund wirft allerdings die Frage auf, warum gerade Planungsnovizinnen und -novizen an diesem wichtigen Prinzip scheitern. 
Neben den mangelnden Planungserfahrungen von Novizinnen und Novizen (Wilson 2016) oder der Linearität ihres Planungsdenkens (Westerman 1991), die zum Teil durch die theoretischen Annahmen von einzelnen Planungsmodellen selbst befördert wird (John 2006), hängt ein entscheidender Grund für dieses Scheitern mit der Komplexität der Unterrichtsplanungsaufgabe zusammen (Gassmann 2013; Mutton, Hagger, und Burn 2011; Wilson 2016), auf die viele der Komplexitätsdimensionen zutreffen, die in integrativen Ansätzen zur Aufgabenkomplexität dargestellt werden (P. Liu und Li 2012). Eine davon betrifft die Interdependenz der Entscheidungen: Die Planenden müssen unter dem insgesamt deutlichen Handlungsdruck des Unterrichts (Wahl 1991) eine grosse Anzahl von unterschiedlichen - und potenziell widersprüchlichen (Stender 2014; Schulz 1972) - Planungselementen überblicken, um beispielsweise Lernvoraussetzung durch adaptive Medienentscheidungen stimmig zu berücksichtigen (König, Buchholtz, und Dohmen 2015; Rey et al. 2018; Zierer und Seel 2012). Diese Komplexität der Planungsaufgabe, die aus dem interdependenten Entscheiden erwächst, stellt hohe kognitive Anforderungen, weil sie auf das kapazitätsbegrenzte Arbeitsgedächtnis als bedeutsamen limitierenden Faktor für wechselseitige Planungsentscheidungen trifft.

\section{Die kognitive Belastung beim Planen als möglicher Grund für die Utopie interdependenten Medienentscheidens}

Eine mögliche Erklärung dafür, warum das Treffen interdependenter Medienentscheidungen als komplexe Planungsaufgabe vor dem Hintergrund des kapazitätsbegrenzten Arbeitsgedächtnisses kognitiv so beanspruchend ist, liefert die Cognitive Load-Theorie (CLT) (Sweller 1988; Sweller, van Merriënboer, und Paas 1998, 2019). In dieser Instruktionsdesigntheorie, die auch eine wichtige Grundlage multimedialer Lerntheorien (Mayer 2005, 2009) darstellt, wird der Fokus auf das Zusammenspiel von Arbeits- und Langzeitgedächtnis bei der Informationsverarbeitung gelegt (Sweller, van Merriënboer, und Paas 2019, 1998).

Im Kern bezieht sich die CLT auf die Erkenntnis, dass das Arbeitsgedächtnis im Unterschied zum - zumindest theoretisch - unbegrenzten Langzeitgedächtnis eine streng limitierte Kapazität hat (Cowan 2001; Miller 1956). Gerade diese Kapazitätsbegrenzung ist vor dem Hintergrund der herausragenden Funktion des Arbeitsgedächtnisses bedeutsam, Informationen temporär zu speichern, sie zu manipulieren und sie zur Konsolidierung mit bestehenden Informationen aus dem Langzeitgedächtnis zu verknüpfen (Kalyuga et al. 2003). Kritisch wird diese Begrenzung insbesondere bei neuen, bis dato ungelernten Informationen, die viel Kapazität im Arbeitsgedächtnis beanspruchen. Für bekannte Informationen, die bereits im Langzeitgedächtnis mit anderen Informationen zu sogenannten Chunks verbunden wurden, wiegt die Kapazitätsbegrenzung weniger schwer, da das Arbeitsgedächtnis Chunks mit beliebig vielen Informationen als einzelne Elemente behandelt. 
Die Kapazität des Arbeitsgedächtnisses, so nimmt die CLT an, kann mehr oder weniger gut genutzt werden, je nachdem, wie stark das Arbeitsgedächtnis bei der Informationsverarbeitung belastet ist (Sweller, van Merriënboer, und Paas 1998, 2019), wobei Sweller et al. mit der intrinsischen, extrinsischen und germanen kognitiven Belastung drei potenzielle Belastungsarten unterscheiden:

- Die intrinsische kognitive Belastung resultiert zum einen aus der Anzahl der zu speichernden und zu verarbeitenden Informationselemente, zum anderen aus deren Interaktivität (Sweller und Chandler 1994). Der Grad der kognitiven Belastung, den diese Elemente und ihre Interaktivität im Arbeitsgedächtnis verursachen, variiert dabei über das Vorwissen (Sweller, van Merriënboer, und Paas 2019; Kalyuga et al. 2003), also den im Langzeitgedächtnis abgelegten Wissenselementen und ihrer hierarchischen Verknüpfungen. Je stärker die einzelnen Elemente des Sachverhalts, der verarbeitet werden soll, miteinander interagieren (die CLT spricht von einer hohen Elementinteraktivität; Sweller und Chandler 1994), und je geringer das Vorwissen ist, desto mehr Elemente müssen simultan im Arbeitsgedächtnis gehalten und verarbeitet werden bzw. desto höher ist die intrinsische kognitive Belastung (van Merriënboer und Sweller 2005; Paas et al. 2003).

- Die extrinsische kognitive Belastung umfasst dagegen den von der inhärenten Komplexität des Gegenstandes unabhängigen Belastungsanteil des Arbeitsgedächtnisses, der von aussen unnötigerweise verursacht wird. Insbesondere suboptimale instruktionale Zugänge (z. B. Redundanzeffekt) und mediale Darstellungsweisen (z. B. Split-Attention-Effekt) bürden oftmals unnötige kognitive Belastungen bei der Informationsverarbeitung auf (Sweller, van Merriënboer, und Paas 2019).

- Die germane kognitive Belastung hängt direkt mit der Informationsverarbeitung zusammen. Gegenwärtig nehmen Sweller, van Merriënboer, und Paas $(2019,264)$ an, dass die germane kognitive Belastung eine Umverteilungsfunktion von extrinsischen zu intrinsischen Aspekten der Aufgabe wahrnimmt und keinen eigenständigen und zu addierenden dritten Belastungsanteil darstellt.

Diese Annahmen zur kognitiven Belastung des kapazitätsbegrenzten Arbeitsgedächtnisses, insbesondere zur hohen intrinsischen Belastung durch eine komplexe Aufgabe, lassen sich auf die Planungsaufgabe des Treffens interdependeter Medienentscheidungen übertragen (Schrader und Schöb 2016): Müssen bei der Begründung der Medienentscheidungen im Zusammenhang mit den weiteren Planungsbedingungen und -entscheidungen viele Planungselemente und deren Interaktionen gleichzeitig berücksichtigt und im Arbeitsgedächtnis aufrechterhalten werden (hohe Elementinteraktivität), dann sorgt dies für eine hohe intrinsische Belastung, die bis zu einer möglichen Überlastung des Arbeitsgedächtnisses führen kann (Sweller und Chandler 1994; Schrader und Schöb 2016). Dabei ist die Komplexität der Aufgabe 
interdependenten Medienentscheidens nicht absolut, sondern relativ zu verstehen, weil sie von dem individuellen Planungsvorwissen abhängt. Gerade Novizinnen und Novizen verfügen über dieses Vorwissen noch nicht, weshalb ihnen die Belastung ihres Arbeitsgedächtnisses besondere Schwierigkeiten mit den Wechselwirkungen von Planungsentscheidungen bereitet.

Auf der Grundlage dieser kognitionspsychologischen Interpretation der Planungsaufgabe interdependenten Entscheidens lässt sich erklären, warum Planungsnovizinnen und -novizen anfangs erst einen Planungsbereich nach dem anderen abarbeiten können und warum das Prinzip der Interdependenz für sie utopisch zu sein scheint. Denn ihnen stehen weder für interdependentes Medienentscheiden noch für die Prozeduralisierung und Konditionalisierung (Tuning) der Planungsfähigkeit (Anderson 1983) kognitive Kapazitäten zur Verfügung. Mit dieser ernüchternden Erklärung geht allerdings die Frage einher, ob dieses gut begründbare mediendidaktische Planungsprinzip und damit der Versuch, eine optimale Strukturqualität von mediengestützten Lernangeboten anzustreben, tatsächlich eine Utopie darstellt. Schon jetzt lässt sich diese Frage verneinen, denn die Fähigkeit von Expertinnen und Experten, interdependente Planungsentscheidungen zu treffen, spricht dagegen, die Utopie-Hypothese als Gedankenspiel aufrechtzuerhalten. Offenbar ist es vielmehr notwendig, genauer der Annahme der nicht änderbaren, hohen intrinsischen kognitiven Belastung der Planungsaufgabe nachzugehen, von der es abzuhängen scheint, ob auch Planungsnovizinnen und -novizen ohne vorhandene Expertise erfolgreich in das interdependente Planungsentscheiden eingeführt werden können.

\section{Die Änderbarkeit der intrinsischen kognitiven Belastung der Planungsaufgabe als Voraussetzung für interdependentes Medienentscheiden}

In der Historie der CLT wurde zunächst angenommen, dass die intrinsische kognitive Belastung nicht durch Veränderungen der Aufgabenstellungen beeinflusst werden könne (Sweller, van Merriënboer, und Paas 1998). Diese Sichtweise führt jedoch zu einem Paradoxon: Eine Aufgabe kann aus so vielen inhärent komplexen Informationsbausteinen mit hoher Elementinteraktivität bestehen, dass bereits die intrinsische kognitive Belastung an sich die Arbeitsgedächtniskapazität übersteigen müsste (Pollock, Chandler, und Sweller 2002). Dann aber wäre unklar, wie bzw. ob unter solchen Umständen Informationen überhaupt verarbeitet werden könnten (van Merriënboer und Sweller 2005). Pollock, Chandler und Sweller $(2002,64)$ schlussfolgerten deshalb aus diesem Paradoxon, dass eine Beeinflussung der intrinsischen kognitiven Belastung durch Veränderungen der Aufgabenstellungen sehr wohl möglich wäre: Novizinnen und Novizen könnten sich lediglich mit Teilelementen einer Aufgabe losgelöst von anderen Teilelementen beschäftigen, ohne die Aufgabe in ihrer Gesamtheit zu verstehen. Entsprechend konnten die Autoren zeigen, dass der 
Lernerfolg von Novizinnen und Novizen - erwartungsgemäss aber nicht bei Expertinnen und Experten - höher ist, wenn die Teilelemente einer Aufgabe zunächst isoliert bearbeitet werden (isolated-elements procedure), sodass die Gesamtaufgabe noch nicht direkt und erst durch zunehmende Berücksichtigung der Zahl der Elemente und ihrer Interaktionen in den Blick gerät, als wenn direkt die Gesamtaufgabe angegangen wird.

Ayres (2006) repliziert und erweitert diese Befunde: Unter Anwendung der isolated-elements procedure sinken Fehlerraten und kognitive Belastung und es lässt sich ein Expertise Reversal-Effekt nachweisen. Gerjets, Scheiter und Catrambone (2004) systematisieren weitere Methoden zur Reduktion von intrinsischer Belastung. Für example-based learning weisen sie in fünf Studien für mathematische Probleme im Vergleich mit molaren, ganzheitlichen Beispielen die positiven Effekte der Verwendung modularer Beispiele nach, bei denen zunächst auf bestimmte strukturelle Charakteristika einer Aufgabe oder die Notwendigkeit mehrerer simultaner Lösungsschritte verzichtet wird.

Zur Veränderbarkeit von intrinsischer kognitiver Belastung stellen van Merriënboer und Sweller (2005) deshalb zusammenfassend fest, dass Befunde wie die von Pollock, Chandler und Sweller (2002) nicht die Validität der ursprünglichen Annahme der Unveränderbarkeit des intrinsischen Loads einer Aufgabe in der CLT untergraben (Sweller, van Merriënboer, und Paas 1998), denn die Massnahme der Reduktion von intrinsischer Belastung geht gleichzeitig mit dem verringerten Verständnis und letztendlich mit einer Veränderung der Aufgabe selbst einher. Novizinnen und Novizen müssten deshalb nach der temporären Entfernung von Elementinteraktivität oder der anfänglich seriellen Verarbeitung von einzelnen Informationen im weiteren Lernprozess trotzdem mit dem Sachverhalt in seiner Gesamtheit und Komplexität konfrontiert werden, die für das Verständnis der Aufgabe notwendig sind (van Merriënboer und Sweller 2005).

Im Sinne dieser Möglichkeit der Beeinflussung der intrinsischen kognitiven Belastung zeigt sich auch das scheinbar utopische Prinzip interdependenten Medienentscheidens in einem anderen Licht. Sollte sich eine Strategie finden lassen, die Novizinnen und Novizen durch eine Zergliederung der Unterrichtsplanungsaufgabe und die sukzessive Erhöhung der Komplexität in das Treffen interdependenter Medienentscheidungen einführt, könnte das Prinzip der Interdependenz auch für Novizinnen und Novizen realisierbar werden. 
5. Der Einsatz von Planungssoftware zur Reduktion der intrinsischen kognitiven Belastung beim interdependenten Medienentscheiden

Eine vielversprechende Möglichkeit zur Veränderung von intrinsischer kognitiver Belastung besteht in der Verwendung von didaktisch sinnvoll eingesetzten (softwaregestützten) Lerntechnologien (Ross, Morrison, und Lowther 2010), deren positive Effekte inzwischen meta-analytisch sowohl für den schulischen Unterricht (Tamim et al. 2011) als auch für den Unterricht in Hochschulen (Schmid et al. 2014) bestätigt sind. Auch zur Unterstützung der Unterrichtsplanung liegt ein grosses Angebot solcher Technologien mit unterschiedlichen Funktionen vor (Prieto et al. 2013). Dabei zielen einige dieser Tools ausdrücklich auf die Reduktion von kognitiver Belastung. So verweist zuletzt Strickroth (2019) im Zuge seiner Konzeptentwicklung für die Anwendung PLATON in einer systematischen Übersicht über elf Planungssysteme beispielhaft auf drei Anwendungen, die diese Reduktion bezwecken sollen, nämlich das Instructional Planning Assistant System (IPAS; T.C. Liu und Juang 2002), das Inquiry in Motion Dynamic Lesson Planning Tool (IIM; Sloop et al. 2014) und das Smart Lesson Planning System (SmartLP; Saad, Chung, und Dawson 2014). Diese Systeme unterliegen jedoch gewissen Einschränkungen, weil weder explizit angehende Lehrkräfte noch Lernprozesse zur Bewältigung der komplexen Unterrichtsplanungsaufgabe fokussiert werden. Stattdessen setzen diese Systeme auf eine effizientere Unterrichtsplanung im Berufsalltag von Lehrkräften durch (webbasierte) Vorlagen und den Zugriff auf eigene und fremde Pläne; und selbst wenn - wie etwa am Beispiel von IPAS deutlich wird - kognitionspsychologische Überlegungen aufgegriffen werden, verbleiben diese Überlegungen auf einer abstrakten Ebene, weil lediglich postuliert wird, dass die kognitive Belastung mithilfe von Scaffolding reduziert werde (T.C. Liu und Juang 2002). In der Evaluation dieser Software spielt die tatsächliche Reduktion von kognitiver Belastung aber keine Rolle (T.C. Liu 2005).

Über diese Systeme hinaus hebt die quasi-experimentell untersuchte Kursplanungs-App von Schrader und Schöb (2016) explizit auf die Reduktion der kognitiven Belastung beim interdependenten Planungsentscheiden ab. Allerdings bezieht sich diese App innerhalb eines vorstrukturierten Planungsrahmens mit vorgegebenen Entscheidungselementen nur auf Unterrichtseinstiege in Lernsettings der Erwachsenen- und Weiterbildung.

Insgesamt scheint es also eine höhere Anzahl an unterstützenden Planungsanwendungen zu geben, die im Rahmen ihrer jeweiligen Kontexte und Ziele chancenreiche Optionen der Planungsunterstützung darstellen. Eine Anwendung, die gezielt die Manipulation von hoher intrinsischer Arbeitsgedächtnisbelastung beim Erlernen interdependenten Entscheidens von Planungsnovizinnen und -novizen fokussiert, gibt es bisher aber noch nicht. Dieses Desiderat war der Anlass dafür, eine eigene Planungssoftware auf der Grundlage der CLT zu entwickeln, die Novizinnen und 
Novizen gezielt bei ihren ersten Unterrichtsplanungsversuchen durch die Reduktion der intrinsischen kognitiven Belastung beim Treffen interdependenter Medienentscheidungen begleiten soll.

\section{Ein Plug-in für Stud.IP als Lerngelegenheit zum interdependenten Medienentscheiden}

Im Zuge des universitären Projektes DU - Digitales Unterrichtscoaching im Bachelor Combined Studies mit Lehramtsoption (ein Kooperationsprojekt an der Universität Vechta unter Leitung des Arbeitsbereichs Schulpädagogik und Allgemeine Didaktik mit Beteiligung der Fachdidaktiken Geographie, Mathematik und Sport, gefördert durch Qualität Plus, Programm für gute Lehre in Niedersachsen des Niedersächsischen Ministeriums für Wissenschaft und Kultur, und unterstützt durch den ELAN e.V.) wurde eine Anwendung als Plug-in für die Lernplattform Stud.IP (Studienbegleitender Internetsupport von Präsenzlehre) entwickelt, die an dieser Universität genutzt wird. Das Plug-in ist für angehende Lehrkräfte gedacht und soll in Seminaren eingesetzt werden können, die in die Unterrichtsplanung einführen und auf (unbegleitete) Schulpraktika vorbereiten. Im Mittelpunkt der Anwendung stehen ausführliche Strukturplanungen von Unterricht, die der obigen Optimierungsidee folgen, und damit unter anderem das begründete Treffen von Medienentscheidungen im Gefüge von sechs Planungsbereichen (situative Voraussetzungen, individuelle Voraussetzungen, Intentionalität, Inhalt, Methodik und Medien), die eine aktuelle Adaption des Entscheidungsmodells der Berliner Didaktik darstellen.

Das Plug-in ermöglicht die Anlage von beliebig vielen schriftlichen Unterrichtsentwürfen. Auf der Übersichtsseite eines jeden Plans werden die sechs Planungsbereiche abgebildet und durch Icons symbolisiert (Abb. 1). Diese Planungsbereiche können in selbst gewählter Reihenfolge bearbeitet werden. Innerhalb der Planungsbereiche können in Eigenregie Felder zu bestimmten Aspekten angelegt werden, z. B. im Bereich individuelle Voraussetzungen $u$. a., zum Vorwissen, Interesse oder Lernund Arbeitsverhalten (Abb. 2) und im Bereich Inhalt zur Sachanalyse oder zur Didaktischen Analyse (die sich wiederum weiter aufgliedern lässt). Jedes dieser Freifelder mit Texteditor kann mit fortwährend änderbaren Notizen befüllt werden, die sich auf Wunsch exportieren lassen. Zu jedem der sechs Planungsbereiche und ihren einzelnen Aspekten liefert die Anwendung Informationsbausteine mit kurzen Definitionen und Erläuterungen am rechten Seitenrand. 


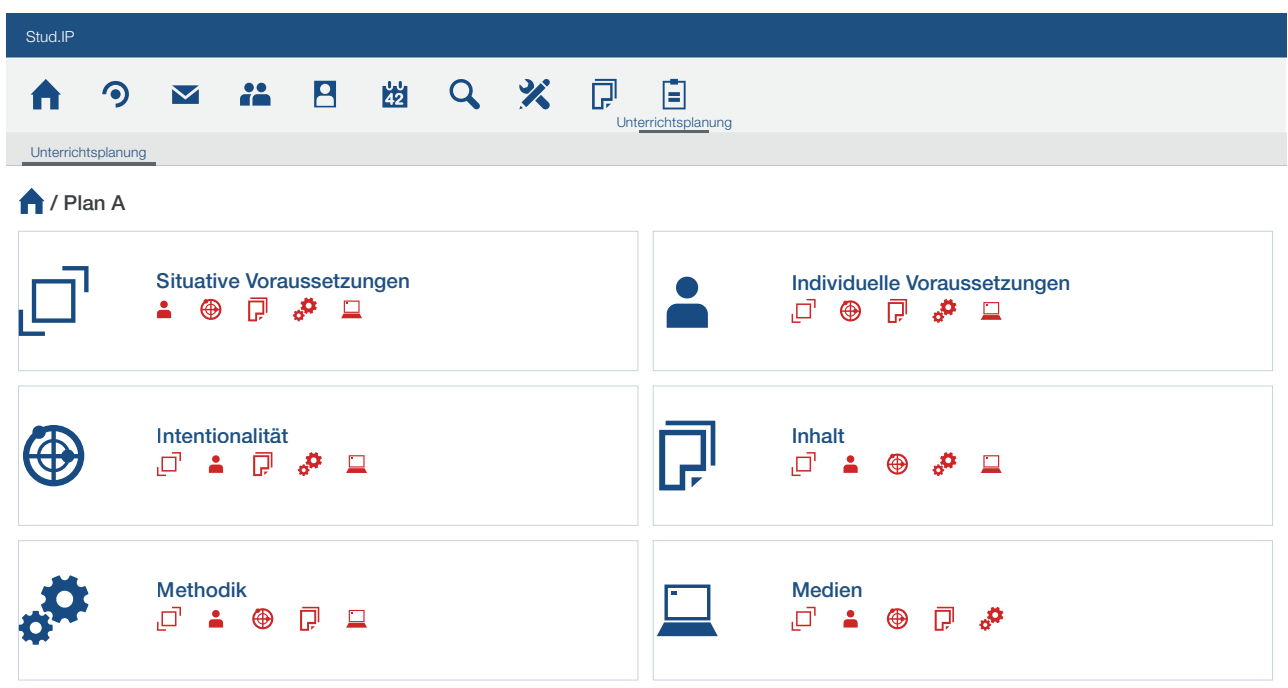

Abb. 1.: Die sechs Planungsbereiche des Plug-ins Unterrichtsplanung in Stud.IP.

Mit der Differenzierung der Planungsbereiche samt -aspekten und den Informationsbausteinen erfüllt das Plug-in ähnliche Funktionen wie die meisten anderen Unterrichtsplanungstechnologien. Zunächst ersetzt es durch diese Funktionen das Medium, in dem in der Regel nicht-planungssoftwarebasierte Planungsversuche unternommen und verschriftlicht werden. Dabei vereinfacht das Plug-in diese Verschriftlichung durch eine bessere Übersichtlichkeit im Vergleich mit herkömmlichen Texteditoren und eine höhere Flexibilität bei der Bearbeitung vorgegebener, erläuterter Planungsbereiche, innerhalb derer die Planungsentscheidungen getroffen und ausgegeben werden können.

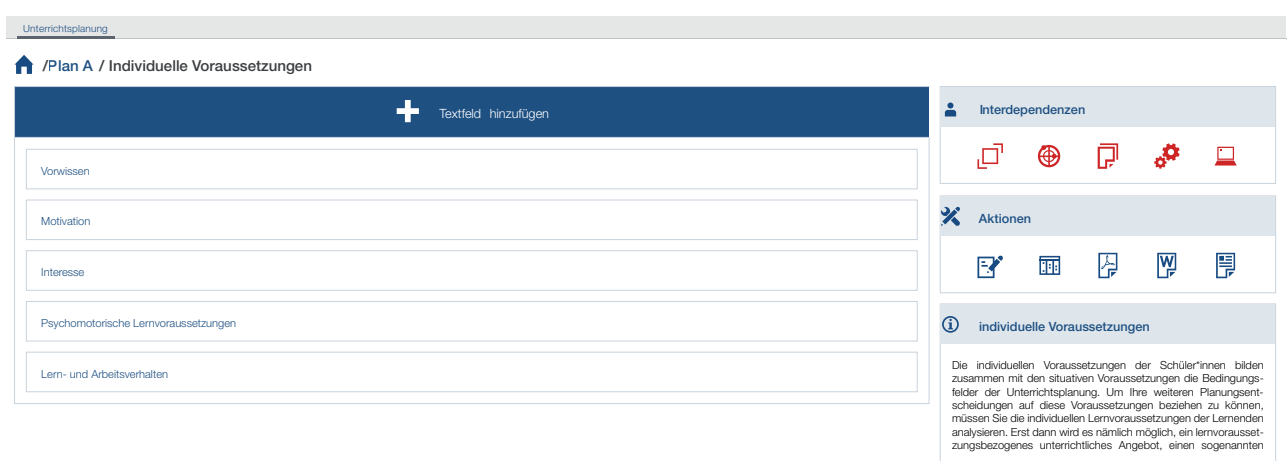

Abb. 2.: Ausschnitt aus dem Planungsbereich individuelle Voraussetzungen mit einzelnen Planungselementen, der Selbstkontrollmöglichkeit berücksichtigter Interdependenzen und Informationsbausteinen.

Über diese Vereinfachung hinaus besteht die zentrale Funktion dieses Plug-ins darin, ein iconbasiertes, strukturiertes Unterstützungssystem für interdependente Planungsentscheidungen zur Reduktion der intrinsischen kognitiven Belastung 
anzubieten (im Sinne des SAMR-Modells eine Neugestaltung der Planungsaufgabe als Modification [Puentedura 2006]). In jedem der sechs Planungsbereiche findet sich an gleicher Stelle eine Anzeige der jeweils anderen fünf Bereiche, dargestellt durch die entsprechenden Icons (Abb. 2, rechts oben). Diese können eingefärbt werden, je nachdem, ob die Entscheidungen im gerade bearbeiteten Planungsbereich kohärent auf bereits getroffene Entscheidungen in den anderen Bereichen abgestimmt wurden. Diese Ikonographie zieht sich nicht nur durch die Planungsbereiche selbst: Auch in der Planübersicht über alle sechs Bereiche werden die Symbole eingefärbt dargestellt und verweisen in einem Ampelsystem auf bisher unbeachtete (rot), einseitig mitgedachte (gelb) und vollständig berücksichtigte (grün) Zusammenhänge (Abb. 3). Dieses Ampelsystem bietet Anwenderinnen und Anwendern die Möglichkeit, die zu verknüpfenden und bereits verknüpften Planungsentscheidungen gemäss dem Prinzip der Interdependenz im Blick zu behalten.

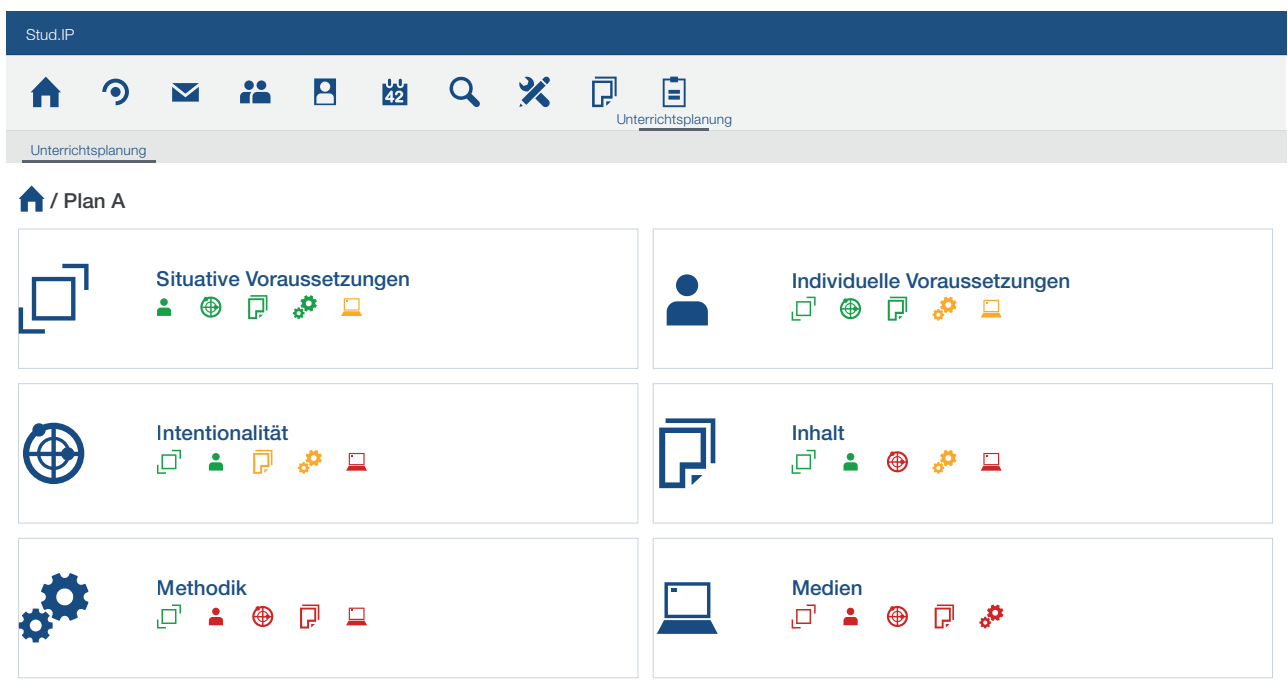

Abb. 3.: Ein Beispiel für markierte Interdependenzsymbole in den sechs Planungsbereichen.

Mithilfe dieses Systems wird versucht, die hohe Komplexität der Unterrichtsplanungsaufgabe und die damit einhergehende hohe intrinsische Belastung des Arbeitsgedächtnisses folgendermassen zu manipulieren: Die Anzeige der anderen Planungsbereiche macht Anwenderinnen und Anwendern bilaterale Entscheidungsprozesse bewusst, indem ein bestimmter Zusammenhang zwischen zwei Planungsbereichen - dem gerade bearbeiteten und einem der fünf durch Icons symbolisierten - aktiviert und in den Blick genommen wird, um für diesen Zusammenhang eine einzelne Entscheidung zu fällen. Dabei lautet die Leitfrage, ob dieser andere Bereich bei der Entscheidung im aktuellen Planungsbereich berücksichtigt wurde, oder aber ob diese Entscheidungen losgelöst von Planungsentscheidungen im anderen Bereich gefällt wurden. Die Unterrichtsplanungsaufgabe selbst wird durch dieses 
Unterstützungssystem in viele Teilaufgaben aufgelöst, die flexibel sequenziell abgearbeitet werden können, ohne dadurch die Dynamik des Planungsdenkens in einer Linearität eines Planungsalgorithmus aufzulösen (eine Art des part-whole sequencing, Gerjets, Scheiter, und Catrambone 2004). Es wird angenommen, dass dadurch die Anzahl der Elemente, die während einer Planungsentscheidung simultan im Arbeitsgedächtnis aufrechterhalten werden müssen, verringert wird.

Mit dieser bewussten Lenkung der Aufmerksamkeit durch Zergliederung der komplexen Planungsaufgabe in einzelne bilaterale Entscheidungsprozesse sollen Planungsnovizinnen und -novizen beim Erkennen von Wechselwirkungen zwischen Planungsentscheidungen, beim Treffen interdependenter Medienentscheidungen für mediengestützte Lernangebote und bei der Reflexion der Kohärenz dieser Entscheidungen gefördert werden. Dabei lässt die Verringerung der intrinsischen kognitiven Belastung des Arbeitsgedächtnisses ein verbessertes selbständiges Erlernen des Treffens interdependenter Planungsentscheidungen erhoffen: Die Arbeitsgedächtniskapazitäten, die durch diese Verringerung befreit werden, können dann zur Übertragung des Planungswissens in das Langzeitgedächtnis und zur Ausbildung von Automatismen interdependenten Entscheidens bei zukünftigen Unterrichtsplanungen nutzbar gemacht werden. Der empirische Nachweis der Effektivität dieser technologiebasierten Lerngelegenheit, die auf Basis der CLT entwickelt wurde, sowohl hinsichtlich der Arbeitsgedächtnisentlastung als auch hinsichtlich der Förderung interdependenten Planens, steht allerdings noch aus.

\section{Diskussion}

Im vorliegenden Beitrag wurde versucht, die Idee der Optimierung während der Unterrichtsplanung mediendidaktisch zu konkretisieren: Medienentscheidungen zu mediengestützten Lernangeboten sind dann optimal, wenn sie im Verhältnis der widerspruchsfreien Wechselwirkung (Interdependenz) zu allen weiteren Planungsentscheidungen und -voraussetzungen stehen und sich die Strukturplanung dieser Lerngelegenheiten als kohärent erweist. Die Legitimation dieser Optimierungsidee wurde dabei in empirischen Befunden zum lernwirksamen Einsatz von Medien gesehen, die ihr Potenzial erst bei einer sinnvollen didaktischen Einbettung entfalten können. Dabei ist diese Idee relativ zu verstehen, weil sich erst im fortlaufenden Kreislauf von Planung, Durchführung und Analyse zeigt, ob die angenommene Kohärenz eines Unterrichtsentwurfs auch tatsächlich zur Lernzielerreichung beigetragen hat.

Diese mediendidaktische Optimierungsidee böte selbst genügend Anlass für eine kritische Analyse, weil die Unterrichtsplanung bei einer überstrengen Auslegung des Prinzips der Interdependenz zumindest der potenziellen Gefahr unterliegen könnte, zu einem unabschliessbaren und unproduktiven Prozess zu verkommen, der sich ähnlich wie bei der Paradoxie der Schaffung von Datenbedarf durch Datensammlung 
(Thompson und Sellar 2018) - in einer Iteration von neuen Fragestellungen bei getroffenen Planungsentscheidungen verlieren könnte. In diesem Beitrag wurde aber eine positivere Sicht auf diese mediendidaktische Idee eingenommen und stattdessen gefragt, ob diese Idee, obwohl berechtigt, nicht vielmehr utopisch sei, weil sie an den empirisch gut belegten Problemen von Novizinnen und Novizen scheitert, Wechselwirkungen zwischen ihren Planungsentscheidungen zu berücksichtigen. Dieser Utopie-Hypothese wurde zunächst genauer nachgegangen, indem die mediendidaktische um eine kognitionspsychologische Perspektive erweitert wurde, aus der heraus sich die Komplexität interdependenten Medienentscheidens als eine zu hohe intrinsische kognitive Belastung vor allem für Planungsnovizinnen und -novizen erweist. Ob diese hohe Belastung allerdings rechtfertige, die Utopie-Hypothese aufrechtzuerhalten, wurde anschliessend unter der Frage diskutiert, inwiefern diese Belastung durch eine Veränderung der Aufgabenstellung beinflussbar ist. Schliesslich wurde eine technologiebasierte Lerngelegenheit vorgestellt, die Planungsnovizinnen und -novizen im Rahmen der in der Literatur aufgezeigten Möglichkeiten zur Veränderung dieser Belastung dabei unterstützen soll, interdependentes Medienentscheiden bei der Unterrichtsplanung zu erlernen: Dieses Plug-in beruht auf der Annahme, dass die Reduktion von intrinsischer kognitiver Belastung durch die Unterteilung der komplexen Planungsaufgabe in bilaterale Entscheidungszusammenhänge Kapazität im Arbeitsgedächtnis freiwerden lässt, die die Speicherung von Planungswissen im Langzeitgedächtnis und eine Automatisierung der Verknüpfung von Planungsentscheidung bis hin zum Gesamtzusammenhang der Bewältigung der Planungsaufgabe begünstigt.

Das mediendidaktische Verständnis von interdependenter Unterrichtsplanung, in dem in diesem Beitrag letztendlich eine bedeutende Voraussetzung für das lernwirksame mediendidaktische Handeln im Unterricht gesehen wird, ist durch eine Idee von Optimierung gekennzeichnet, die eng mit der gestaltungsorientierten Mediendidaktik und ihrer Frage, «wie Potenziale von digitalen Medien für das Lernen und Lehren eingelöst werden können» (Kerres 2018, 83), sowie der mediendidaktischen Hoffnung auf «Steigerung der Effektivität und/oder der Effizienz des Lernens» (Kerres und Kalz 2003, 413) verknüpft ist.

Diese Optimierungsidee, die in deutlicher Nähe zur Optimierung als übergeordnetem Leitmotiv der empirischen Bildungsforschung steht (Cortina 2020) und zum Beispiel auch bei dem vergleichbaren Vorgehen der Entwicklung einer CLT- und technologiebasierten Unterrichtsplanungslerngelegenheit bei Schrader und Schöb (2016) so ausgelegt wird, lässt sich allerdings selbst hinterfragen. So ist es gemäss dieser Idee vor allem die Lehrkraft, die verantwortlich für die Unterrichtsplanung und damit die optimale Vorbereitung des Unterrichts durch Grundlegung seiner Strukturqualität zeichnet. Schon Schulz als früheres Mitglied des Berliner Kreises zweifelt aber in seiner kritisch orientierten Hamburger Didaktik die Exklusivität 
dieses Optimierungsanspruchs bei der Lehrkraft an. In dieser Didaktik fordert er, «daß auch der Prozeß der Unterrichtsplanung letztlich eine Interaktion zwischen den Unterrichtsteilnehmern sein sollte und damit Teil des Unterrichts selbst» (Schulz 1981, 12). Mit dieser Forderung erweitert Schulz die allgemeindidaktische Annahme der Optimierung. Die Unterrichtsplanung ist nicht mehr nur dann optimal, wenn sie einen kohärenten Unterrichtsentwurf als Folge interdependenten Entscheidens hervorbringt, sondern auch dann, wenn ein solcher - allerdings immer noch in sich stimmiger - Entwurf im Unterricht gemeinsam mit den Schülerinnen und Schülern in einer dialogischen (Schulz 1981, 5) bzw. partizipatorischen Planung (Schulz 1981, 13) zustande kommt.

Auch in der Mediendidaktik, die schon in der gestaltungsorientierten Position den Gedanken der Partizipation aufgreift, wird dieses erweiterte Verständnis von Optimierung stark gemacht. Gegenwärtig bringt die partizipative Mediendidaktik in aller Deutlichkeit auf den Punkt, dass es ihr

«im Sinne eines engeren Begriffs von Bildungsmedien nicht in erster Linie um die Gestaltung von in sich eher geschlossenen oder für sich stehenden Medienangeboten, sondern [...] in einem weiteren Medienverständnis primär um die Gestaltung von komplexen personalen Lernumgebungen» (Mayrberger 2019, 120)

geht. Ein solch erweitertes Medienverständnis mit seiner Vorstellung von der Komplexität personaler Lernumgebungen geht nicht mehr allein in einer lehrkraftzentrierten Optimierungsidee der Steigerung der Lernwirksamkeit durch interdependente Medienentscheidungen auf, bei der sich die Frage nach der Verwirklichung dieser Idee unter anderem aus kognitionspsychologischer Perspektive stellt. Ein solches erweitertes Verständnis der Optimierungsidee hat vielmehr zur Folge, «dass alle mediendidaktischen [...] Planungsentscheidungen die Schaffung, Gestaltung und Reflexion eines gemeinsamen Partizipationsraums zum Ziel haben» (Mayrberger 2020, 73). Dieses Ziel, das sowohl Konsequenzen für die Planungszuständigkeiten als auch -entscheidungen hat, ist allerdings noch nicht Gegenstand der vorgestellten Planungstechnologie. Vielleicht wäre also die kritische Weiterführung der aufgegriffenen mediendidaktischen Optimierungsidee in Richtung Partizipation eine mögliche Perspektive, um auch die Entwicklung von förderlichen Planungstechnologien zur Unterstützung von Planungsnovizinnen und -novizen voranzutreiben. Denn dass die optimale Planung von mediengestützten Lernangeboten noch komplexer ist als in der Berliner Didaktik beschrieben und mit zunehmender Komplexität noch höhere Anforderungen an die Planenden stellt, scheint sicher und ein fortwährender Anlass zu sein, Planungsnovizinnen und -novizen auf ihrem Weg zu Expertinnen und Experten bestmöglich zu unterstützen. 


\section{Literatur}

Anderson, John R. 1983. The architecture of cognition. Cambridge, Mass.: Harvard University Press.

Ayres, Paul. 2006. «Impact of reducing intrinsic cognitive load on learning in a mathematical domain». Applied Cognitive Psychology 20 (3): 287-98. https://doi.org/10.1002/acp.1245.

Balandis, Oswald, und Jürgen Straub. 2018. «Selbstoptimierung und Enhancement». Journal für Psychologie 26 (1): 131-55. https://doi.org/10.30820/8247.09.

Bellmann, Johannes, Marcelo Caruso, und Elke Kleinau. 2020. «Optimierung in Bildung und Erziehung: Einleitende Thesen in den Thementeil». Zeitschrift für Pädagogik 2020 (1): 1-7. https://doi.org/10.3262/ZP2001001.

Bromme, Rainer, und Falk Seeger. 1979. Unterrichtsplanung als Handlungsplanung: Eine psychologische Einführung in die Unterrichtsvorbereitung. Entwicklung praxisorientierter Ausbildungs- und Studienmaterialien für Mathematiklehrer der Sekundarstufe I. Band 1. Königstein/Ts.: Scriptor.

Cortina, Kai S. 2020. «Zur Optimierbarkeit von Lernen und Lehren aus empirischer Sicht». Zeitschrift für Pädagogik 2020 (1): 72-77. https://doi.org/10.3262/ZP2001072.

Cowan, Nelson. 2001. «The magical number 4 in short-term memory: A reconsideration of mental storage capacity». The Behavioral and Brain Sciences 24 (1): 87-185. https://doi. org/10.1017/S0140525X01003922.

de Witt, Claudia, und Thomas Czerwionka. 2013. Mediendidaktik. Bielefeld: W. Bertelsmann Verlag. https://doi.org/10.3278/42/0037w.

Friesen, Norm. 2010. «Lesson planning: Anglo-American perspectives». Bildung und Erziehung 63 (4): 417-30. https://doi.org/10.7788/bue.2010.63.4.417.

Gassmann, Claudia. 2013. Erlebte Aufgabenschwierigkeit bei der Unterrichtsplanung: Eine qualitativ-inhaltsanalytische Studie zu den Praktikumsphasen der universitären Lehrerbildung. Wiesbaden: Springer VS. https://doi.org/10.1007/978-3-658-00243-5.

Gerjets, Peter, Katharina Scheiter, und Richard Catrambone. 2004. «Designing instructional examples to reduce intrinsic cognitive load: Molar versus modular presentation of solution procedures». Instructional Science 32 (1/2): 33-58. https://doi.org/10.1023/ B:TRUC.0000021809.10236.71.

Hattie, John. 2015. Lernen sichtbar machen. Baltmannsweiler: Schneider Verlag Hohengehren.

Heimann, Paul. (1962) 1976. «Didaktik als Theorie und Lehre». In Paul Heimann - Didaktik als Unterrichtswissenschaft, hrsg. von Kersten Reich und Helga Thomas. 1. Aufl. Stuttgart: Klett.

John, Peter D. 2006. «Lesson planning and the studentteacher: Re-thinking the dominant model». Journal of Curriculum Studies 38 (4): 483-98. https://doi.org/10.1080/00220270500363620.

Kalyuga, Slava, Paul Ayres, Paul Chandler, und John Sweller. 2003. «The expertise reversal effect». Educational Psychologist 38 (1): 23-31. https://doi.org/10.1207/S15326985EP3801_4. 
Kerres, Michael. 2000. «Medienentscheidungen in der Unterrichtsplanung Zu Wirkungsargumenten und Begründungen des didaktischen Einsatzes digitaler Medien». Bildung und Erziehung 53 (1): 19-40. https://doi.org/10.7788/bue.2000.53.1.19.

Kerres, Michael. 2005. «Gestaltungsorientierte Mediendidaktik und ihr Verhältnis zur Allgemeinen Didaktik». In Allgemeine Didaktik im Wandel, hrsg. von Peter Stadtfeld und Bernhard Dieckmann, 214-34. Bad Heilbrunn: Klinkhardt.

Kerres, Michael. 2018. Mediendidaktik: Konzeption und Entwicklung digitaler Lernangebote. De Gruyter Studium. Berlin: De Gruyter Oldenbourg. https://doi.org/10.1515/9783110456837.

Kerres, Michael, und Marco Kalz. 2003. «Mediendidaktik in der Lehrerbildung». Beiträge zur Lehrerinnen- und Lehrerbildung 21 (3): 410-21. https://nbn-resolving.org/urn:nbn:de:0111pedocs-135330.

Koeppen, Kim E. 1998. «The experiences of a secondary social studies student teacher: Seeking security by planning for self». Teaching and Teacher Education 14 (4): 401-11. https:// doi.org/10.1016/S0742-051X(97)00047-4.

König, Johannes, Christiane Buchholtz, und Dieter Dohmen. 2015. «Analyse von schriftlichen Unterrichtsplanungen: Empirische Befunde zur didaktischen Adaptivität als Aspekt der Planungskompetenz angehender Lehrkräfte». Zeitschrift für Erziehungswissenschaft 18 (2): 375-404. https://doi.org/10.1007/s11618-015-0625-7.

Kreis, Annelies, und Fritz Staub. 2017. Kollegiales Unterrichtscoaching: Das Instrument zur praxissituierten Unterrichtsentwicklung. Köln: Carl Link Verlag.

Liu, Peng, und Zhizhong Li. 2012. «Task complexity: A review and conceptualization framework». International Journal of Industrial Ergonomics 42 (6): 553-68. https://doi.org/10.1016/j.ergon.2012.09.001.

Liu, Tzu-Chien. 2005. «Web-based cognitive apprenticeship model for improving pre-service teachers' performances and attitudes towards instructional planning: Design and field experiment». Educational Technology \& Society 8 (2): 136-49. https://drive.google.com/open ?id=1U_3VrymA8L6z2mL37ENXn9xTVd3e4xBG.

Liu, Tzu-Chien, und Yih-Ruey Juang. 2002. «IPAS - Teacher's knowledge management platform for teachers professional development». Proceedings of the International Conference on Engineering Education, Manchester, UK, 18. August.

Mayer, Richard E. 2005. "Cognitive theory of multimedia learning». In The Cambridge Handbook of Multimedia Learning, hrsg. von Richard E. Mayer, 31-48. Cambridge: Cambridge University Press.

Mayer, Richard E. 2009. Multimedia Learning. Cambridge: Cambridge University Press.

Mayrberger, Kerstin. 2020. «Partizipative Mediendidaktik. Darstellung von Eckpunkten und Vertiefung des Partizipationsraums als konstituierendes Strukturelement». Zeitschrift MedienPädagogik 17 (Jahrbuch Medienpädagogik): 59-92. https://doi.org /10.21240/mpaed/ jb17/2020.04.26.X.

Mayrberger, Kerstin. 2019. Partizipative Mediendidaktik. Gestaltung der (Hochschul-) Bildung unter den Bedingungen der Digitalisierung. Weinheim: Beltz Juventa. 
Miller, George A. 1956. "The magical number seven, plus or minus two: Some limits on our capacity for processing information». Psychological Review 63 (2): 81-97. https://doi. $\operatorname{org} / 10.1037 / \mathrm{h} 0043158$.

Mutton, Trevor, Hazel Hagger, und Katharine Burn. 2011. «Learning to plan, planning to learn: the developing expertise of beginning teachers». Teachers and Teaching 17 (4): 399-416. https://doi.org/10.1080/13540602.2011.580516.

Paas, Fred G., Juhani E. Tuovinen, Huib Tabbers, und Pascal W. M. van Gerven. 2003. «Cognitive load measurement as a means to advance cognitive load theory». Educational Psychologist 38 (1): 63-71. https://doi.org/10.1207/S15326985EP3801_8.

Petko, Dominik. 2020. Einführung in die Mediendidaktik: Lehren und Lernen mit digitalen Medien. Weinheim: Beltz.

Pollock, Edwina, Paul Chandler, und John Sweller. 2002. «Assimilating complex information». Learning and Instruction 12 (1): 61-86. https://doi.org/10.1016/S0959-4752(01)00016-0.

Prieto, Luis P., Yannis Dimitriadis, Brock Craft, Michael Derntl, Valérie Émin, Mary Katsamani, Diana Laurillard, Elizabeth Masterman, Symeon Retalis, und Eloy Villasclaras. 2013. «Learning design Rashomon II: Exploring one lesson through multiple tools». Research in Learning Technology 21. https://doi.org/10.3402/rlt.v21i0.20057.

Puentedura, Ruben R. 2006. Transformation, technology, and education. http://www.hippasus.com/resources/tte/.

Rey, Thomas, Hendrik Lohse-Bossenz, Albrecht Wacker, und Vera Heyl. 2018. «Adaptive Planungskompetenz bei angehenden Lehrkräften in der zweiten Phase der Lehrerbildung. Befunde einer Pilotierungsstudie aus Baden-Württemberg». heiEDUCATION Journal 1/2: 127-50. https://doi.org/10.17885/HEIUP.HEIED.2018.1-2.23829.

Ross, Steven M., Gary R. Morrison, und Deborah L. Lowther. 2010. «Educational technology research past and present: Balancing rigor and relevance to impact school learning». Contemporary Educational Technology 1 (1): 17-35. https://doi.org/10.30935/cedtech/5959.

Russell, Thomas L. 1999. The no significant difference phenomenon: as reported in 355 research reports, summaries and papers. Raleigh: North Carolina State University.

Saad, Aslina, Paul W. H. Chung, und Christian W. Dawson. 2014. «Effectiveness of a case-based system in lesson planning». Journal of Computer Assisted Learning 30 (5): 408-24. https:// doi.org/10.1111/jcal.12053.

Schmid, Richard F., Robert M. Bernard, Eugene Borokhovski, Rana M. Tamim, Philip C. Abrami, Michael A. Surkes, C. Anne Wade, und Jonathan Woods. 2014. «The effects of technology use in postsecondary education: A meta-analysis of classroom applications». Computers \& Education 72: 271-91. https://doi.org/10.1016/j.compedu.2013.11.002.

Schrader, Josef, und Sabine Schöb. 2016. «Die Planung von Lehr-Lern-Einheiten mit digitalen Medien: Konzepte und Befunde». Zeitschrift für Weiterbildungsforschung 39 (3): 331-47. https://doi.org/10.1007/s40955-016-0078-5.

Schulmeister, Rolf. 2007. Grundlagen hypermedialer Lernsysteme: Theorie - Didaktik - Design. München: Oldenbourg Verlag. https://doi.org/10.1524/9783486594096. 
Schulz, Wolfgang. 1972. «Unterricht - Analyse und Planung». In Unterricht. Analyse und Planung, hrsg. von Paul Heimann, Gunter Otto und Wolfgang Schulz, 13-47. Hannover: Hermann Schroedel Verlag.

Schulz, Wolfgang. 1981. Unterrichtsplanung: Mit Materialien aus Unterrichtsfächern. München: Urban \& Schwarzenberg.

Seifried, Jürgen. 2009. «Unterrichtsplanung von (angehenden) Lehrkräften an kaufmännischen Schulen». Zeitschrift für Berufs- und Wirtschaftspädagogik 105 (2): 179-97. https:// elibrary.steiner-verlag.de/article/99.105010/zbw200902017901.

Sloop, Ben, Bob Horton, Jeff Marshall, und Robbie Higdon. 2014. «Mathematical inquiry: An instructional model and web-based lesson-planning tool for creating, refining, and sharing inquiry-based lessons». MathMate 36: 28-36.

Stender, Anita. 2014. Unterrichtsplanung: Vom Wissen zum Handeln; Theoretische Entwicklung und empirische Überprüfung des Transformationsmodells der Unterrichtsplanung. Studien zum Physik- und Chemielernen 170. Berlin: Logos-Verlag.

Strickroth, Sven. 2019. «PLATON: Developing a graphical lesson planning system for prospective teachers». Education Sciences 9 (4): 254. https://doi.org/10.3390/educsci9040254.

Sweller, John. 1988. "Cognitive load during problem solving: Effects on learning». Cognitive Science 12 (2): 257-285. https://doi.org/10.1207/s15516709cog1202_4.

Sweller, John, und Paul Chandler. 1994. "Why some material is difficult to learn». Cognition and Instruction 12 (3): 185-233. https://doi.org/10.1207/s1532690xci1203_1.

Sweller, John, Jeroen J. G. van Merriënboer, und Fred G. Paas. 1998. «Cognitive architecture and instructional design». Educational Psychology Review 10 (3): 251-96. https://doi. org/10.1023/A:1022193728205.

Sweller, John, Jeroen J. G. van Merriënboer, und Fred G. Paas. 2019. «Cognitive architecture and instructional design: 20 years later». Educational Psychology Review 31 (2): 261-92. https://doi.org/10.1007/s10648-019-09465-5.

Tamim, Rana M., Robert M. Bernard, Eugene Borokhovski, Philip C. Abrami, und Richard F. Schmid. 2011. «What forty years of research says about the impact of technology on learning». Review of Educational Research 81 (1): 4-28. https://doi.org/10.3102/0034654310393361.

Thompson, Greg, und Sam Sellar. 2018. «Datafication, testing events and the outside of thought». Learning, Media and Technology 43 (2): 139-51. https://doi.org/10.1080/17439 884.2018.1444637.

van Merriënboer, Jeroen J. G., und John Sweller. 2005. "Cognitive load theory and complex learning: Recent developments and future directions». Educational Psychology Review 17 (2): 147-77. https://doi.org/10.1007/s10648-005-3951-0.

Wahl, Diethelm. 1991. Handeln unter Druck: Der weite Weg vom Wissen zum Handeln bei Lehrern, Hochschullehrern und Erwachsenenbildnern. Weinheim: Dt. Studien-Verlag. 
Weitzel, Holger, und Robert Blank. 2019. «Peer Coaching und fachdidaktische Unterrichtsplanung - ein Overload?». In Herausforderung Kohärenz: Praxisphasen in der universitären Lehrerbildung: Bildungswissenschaftliche und fachdidaktische Perspektiven, hrsg. von Maria Degeling, Nadine Franken und Stefan Freund, 393-404. Bad Heilbrunn: Verlag Julius Klinkhardt.

Wengert, Hans Gert. 1989. Untersuchungen zur alltäglichen Unterrichtsplanung von Mathematiklehrern: Eine kognitionspsychologische Studie. Europäische Hochschulschriften Reihe 6, Psychologie 275. Frankfurt am Main: Lang.

Westerman, Delores A. 1991. «Expert and novice teacher decision making». Journal of Teacher Education 42 (4): 292-305. https://doi.org/10.1177/002248719104200407.

Wilson, Susanna. 2016. «Developing mathematical content knowledge for teaching: One preservice teacher and her planning». In Opening up mathematics education research (Proceedings of the 39th annual conference of the Mathematics Education Research Group of Australasia), hrsg. von Bruce White, Mohan Chinnappan und Sven Trenholm, 624-31. Adelaide: MERGA.

Zierer, Klaus, und Norbert M. Seel. 2012. «General didactics and instructional design: Eyes like twins: A transatlantic dialogue about similarities and differences, about the past and the future of two sciences of learning and teaching». SpringerPlus 1 (15). https://doi. org/10.1186/2193-1801-1-15. 